\title{
Heterogeneous String Stability of Unidirectionally Interconnected MIMO LTI Systems *
}

\author{
Gábor Rödönyi \\ Systems and Control Laboratory, Institute for Computer Science and Control, Hungarian Academy of Sciences, 1111 Kende \\ u. 13-17 Budapest, Hungary
}

\begin{abstract}
One-dimensional formations of unidirectionally interconnected heterogeneous, multiple-input multiple-output (MIMO) linear time-invariant (LTI) systems are studied in terms of the spatial propagation of initial conditions, disturbances and reference inputs. The proposed mixed frequency-domain and complex state-space formulation of the string leads to the stability theory of switching and uncertain polytopic systems, and so the tools available there can be adopted for the analysis of interconnected systems. It is shown based on this analogy that in certain important cases string stability conditions for homogeneous and heterogeneous interconnected systems coincide. In addition to presenting general string (in)stability conditions, the necessity of introducing the notion of string performance is demonstrated. Special attention is devoted to interconnected rank one systems, i.e., systems whose transfer matrices are of rank one. The topic is motivated by car following problems for which the available analysis tools fail to provide appropriate heterogeneous string stability conditions. A cooperative adaptive cruise control (CACC) example is presented to illustrate the usefulness of the approach.
\end{abstract}

Key words: Vehicle platoons, string stability, switching systems, joint spectral radius, cooperative adaptive cruise control.

\section{Introduction}

A general framework is presented for the study of $1-\mathrm{D}$ formations (strings) of unidirectionally interconnected heterogeneous LTI systems where the ordering of the components is arbitrary. Specifically, spatial and temporal evolution of signals and the notion of string stability are examined for interconnections which are subject to nonzero initial conditions, disturbances and reference inputs, and where the flow of information between the components is unidirectional along the string. Such systems arise in many fields of application, for instance, automated irrigation channels (Soltanian and Cantoni, 2015), supply chains (Huang et al., 2007), harmonic oscillators $\mathrm{Yu}$ et al. (2015), lateral (McAree and Veres, 2016) and longitudinal (Ioannou and Chien, 1993) control of vehicle platoons.

String stability of a string ensures boundedness of signals as they evolve both in time and space. Most of

\footnotetext{
* This work was supported by the János Bolyai Research Scholarship of the Hungarian Academy of Sciences and by the Excellent Center for Vehicle Technology (J3K) No: MTA KEP-4/2017.
}

Email address: rodonyi@sztaki.hu (Gábor Rödönyi). the available analysis and design approaches are applicable only to homogeneous strings where the interconnected components are identical in their dynamics (Swaroop and Hedrick, 1996; Peters et al., 2014). Methods that concern the string stability of heterogeneous unidirectionally interconnected formations, e.g., (Kianfar et al., 2011; Lidstrom et al., 2012; Monteil et al., 2018), are limited to single input single output (SISO) systems and special problems. The most widespread analysis methods for string stability can be classified in view of the applied transformations on the twodimensional (time and space) signal spaces associated with the interconnected systems. (Time,Frequency)and (Frequency,Frequency)-domain analysis methods (Šebek and Hurák, 2011; Knorn, 2013) are based on Z-transform for the spatial sequence of signals and dynamic systems. The approach requires a certain spatial invariance; therefore, it cannot be applied to heterogeneous strings. (Time,Space)-domain approaches are based on infinite dimensional operators (D'Andrea and Dullerud, 2003); (vector) Lyapunov functions (Swaroop and Hedrick, 1996); and the theory of 2-D systems (Knorn, 2013; Soltanian and Cantoni, 2015). Soltanian and Cantoni derived a distributed sufficient condition for heterogeneous string stability (HSS) demanding uniform boundedness in both time and space (Soltanian 
and Cantoni, 2015). A great advantage of their results is that HSS of the string can be achieved by satisfying a set of independent local conditions. Unfortunately, for the vehicle platooning problem presented in this paper that sufficient condition never holds, yet the system may be HSS, see Remark 5. Applying Laplace transformation for each signal and LTI component model leads to the (Frequency, Space)-domain approaches. The most widespread analysis method of this class requires the computation of string stability transfer functions

$$
\Gamma_{i}(s)^{\prime} \triangleq \frac{z_{i}(s)}{z_{0}(s)}, \text { or } \Gamma_{i}(s) \triangleq \frac{z_{i}(s)}{z_{i-1}(s)},
$$

which yield the respective weak $\left(\left\|\Gamma_{i}^{\prime}\right\|_{\infty} \leq 1\right)$ and strong $\left(\left\|\Gamma_{i}\right\|_{\infty} \leq 1\right)$ conditions for string stability with respect to the variable of interest, $z_{i}$ (Shaw and Hedrick, 2007; Naus et al., 2010). These transfer functions depend in general on the properties of multiple components and so the approach requires extensive numerical tests for the analysis in the heterogeneous case and gives no hints on component (re)design (Naus et al., 2010). The choice of the variable of interest also influences the type of string stability, for which there exist no generally valid theoretical explanations.

In this paper we propose a (Frequency, Space)-domain approach where the string is viewed as a parameter dependent complex valued discrete dynamical system. The concept immediately suggests the introduction of the notion of heterogeneous string performance (HSP) which helps resolving the above mentioned problems. The proposed approach has been tested on special SISO problems (Rödönyi, 2018, 2017). In this paper we provide a general framework for developing conditions that guarantee string stability of heterogeneous and multivariable ad hoc strings. The main properties and contributions with respect to existing results are summarized as follows.

(1) The class of systems under consideration is general: (a) heterogeneous strings of MIMO LTI components in arbitrary order; (b) unidirectional interconnection structure involving leader and multiple predecessor following architectures;

(2) Effects of initial conditions, disturbances and reference signals are analyzed in a common framework;

(3) The introduced analysis approach reveals analogies between interconnected systems and switching systems with important conclusions: (a) well developed powerful theoretical and numerical tools appear on the field of interconnected systems (b) classes of systems, for which the conditions for homogeneous and heterogeneous string stability coincide, can be characterized (c) the introduction of the notion of string performance resolves some existing dilemmas in the field

The paper is organized as follows. The problem is formu- lated in Section 2. In Section 3 a state-space like description of interconnected systems is proposed that highlights the difference between the notions of string stability and string performance. General and special string stability and performance conditions are presented in Sections 4 and 5, respectively. Finally, a CACC problem is presented in Section 6, which illustrates some advantages of the proposed approach.

\section{Notations}

$\mathbb{N}, \mathbb{R}, \mathbb{C}, \mathbb{N}_{+}, \mathbb{R}_{>0}$ and $\mathbb{C}^{+}$denote respectively the field of natural, real and complex numbers, the positive integers, the nonnegative real numbers, and the open right complex plane. |.| denotes both the absolute value of a scalar and an appropriate vector norm associated with a finite dimensional vector space. The dimension of vector $x$ is denoted by $\operatorname{dim}(x)$. The transpose of a vector or matrix is denoted by the subscript ${ }^{T}$. The maximum singular value and respectively the spectral radius of a matrix are denoted by $\bar{\sigma}($.$) and \rho($.$) . If \mathcal{A}$ is an object (number/matrix/system/...), then $\mathcal{S}_{\mathcal{A}}$ denotes a set of these objects. Conv denotes convex hull. The joint spectral radius (JSR) of a set of matrices $\mathcal{S}_{\mathcal{A}}$ is defined by $\sigma_{\mathcal{A}} \triangleq \lim _{\ell \rightarrow \infty} \sup \left\{\|M\|^{1 / \ell}, M \in \mathcal{S}_{\mathcal{A}}^{\ell}\right\}$, where $\|$.$\| denote any matrix norm and \mathcal{S}_{\mathcal{A}}^{\ell}$ denotes the set of all matrix products of length $\ell$ with factors from set $\mathcal{S}_{\mathcal{A}}$. Let $\ell_{p}$ denote the space of sequences of vectors $x=\left\{x_{i} \in \mathbb{R}^{\operatorname{dim}\left(x_{i}\right)}, i \in \mathbb{N}\right\}$, with the norm $\|x\|_{p} \triangleq$ $\left(\sum_{i=0}^{\infty}\left|x_{i}\right|^{p}\right)^{1 / p}$ for $p \in[1, \infty)$ and $\|x\|_{\infty} \triangleq \max _{i}\left|x_{i}\right|$ for $p=\infty$. For $p \in[1, \infty]$ the function space $\mathcal{L}_{p}$ denotes $\left\{x:[0, \infty) \mapsto \mathbb{R}^{n}: x\right.$ is measurable and $\|x\|_{p}<$ $\infty\}$, where $\|x\|_{p} \triangleq\left(\int_{0}^{\infty}|x(t)|^{p} d t\right)^{1 / p}$ for $p \in[1, \infty)$ and $\|x\|_{\infty} \triangleq \operatorname{ess}_{\sup _{t \geq 0}}|x(t)|$ for $p=\infty$. Fourier and Laplace transforms of time-domain functions $x(t)$ are denoted by the same symbol with appropriate arguments, $x(j \omega)=\mathcal{F}\{x(t)\}$ and $x(s)=\mathcal{L}\{x(t)\}$ with $\omega \in \mathbb{R}$ and $s \in \mathbb{C}$, respectively. By Parseval's Theorem we have $\|x\|_{2}=\left(\frac{1}{2 \pi} \int_{-\infty}^{\infty}|x(j \omega)|^{2} d \omega\right)^{1 / 2} \cdot \mathcal{H}_{\infty}$ denotes the Hardy space of functions $G: \mathbb{C}^{+} \mapsto \mathbb{C}^{p \times m}$ that are analytic on $\mathbb{C}^{+}$. The norm in this space can be computed as $\|G\|_{\infty}=\operatorname{ess}_{\sup _{\omega \in \mathbb{R}}} \bar{\sigma}(G(j \omega))$. Let $\left(\mathcal{L}_{p}, \ell_{q}\right) \triangleq\left\{x_{i}(t) \in\right.$ $\left.\mathbb{R}^{\operatorname{dim}\left(x_{i}\right)}, i \in \mathbb{N}, t \in \mathbb{R}_{\geq 0},\|x\|_{p, q}<\infty\right\}$ denote the 2-D space of sequences of vector valued functions, which is endowed with the norm $\|x\|_{p, q} \triangleq\left(\sum_{i=0}^{\infty}\left\|x_{i}(.)\right\|_{p}^{q}\right)^{1 / q}$ for $q \in[1, \infty)$ and $\|x\|_{p, \infty}=\sup _{i \geq 0}\left\|x_{i}(.)\right\|_{p}$ for $q=\infty$.

\section{Problem Formulation}

\subsection{Local Component Model}

Leader and multiple predecessor following 1-D formations (strings) of dynamic components are considered in the paper. The leader is represented by a signal $q_{0}(t) \in$ 
$\mathbb{R}^{n_{q}}$, which can be considered as a spatial boundary condition to the string. In some applications $q_{0}$ is interpreted as a reference signal to be asymptotically followed by the other components (e.g., reference position in longitudinal platooning problems (Ioannou and Chien, 1993), water flow-rate in automated irrigation channels (Soltanian and Cantoni, 2015)). The components of the string are denoted by $\Sigma_{i}, i \in \mathbb{N}$, and are described by the following LTI state-space equations

$$
\left[\begin{array}{c}
\dot{x}_{i}(t) \\
\hline q_{i+1}(t) \\
z_{i}(t)
\end{array}\right]=\left[\begin{array}{c|ccc}
A_{i} & B_{i, \mathcal{N}_{i}} & B_{i, 0} & B_{i, d} \\
\hline C_{i, q} & D_{i, \mathcal{N}_{i}, q} & D_{i, 0, q} & D_{i, d, q} \\
C_{i, z} & D_{i, \mathcal{N}_{i}, z} & D_{i, 0, z} & D_{i, d, z}
\end{array}\right]\left[\begin{array}{c}
x_{i}(t) \\
q_{\mathcal{N}_{i}}(t) \\
q_{0}(t) \\
d_{i}(t)
\end{array}\right],
$$

where $x_{i}(t) \in \mathbb{R}^{\operatorname{dim}\left(x_{i}\right)}$ denotes the state vector of the local system with initial condition $x_{i}(0)=x_{i, 0}$. The state space dimensions $\operatorname{dim}\left(x_{i}\right)$ are not necessarily equal for all components. Signals $q_{i}, i \in \mathbb{N}$, are called transmission signals, have equal dimensions $\operatorname{dim}\left(q_{i}\right)=n_{q}$ for all components, and represent the information that is propagating along the string (e.g., vehicle acceleration in platooning problems). They are not necessarily equivalent to the variables of interest (e.g., spacing errors in platooning problems). The variables of interest are called performance outputs, and are denoted by $z_{i}(t) \in \mathbb{R}^{\operatorname{dim}\left(z_{i}\right)}$, $i \in \mathbb{N}$. The dimension of the performance output may differ for each component. Each component $\Sigma_{i}$ is subject to disturbance $d_{i}(t) \in \mathbb{R}^{\operatorname{dim}\left(d_{i}\right)}$ whose dimension may depend on $i$. State space matrices $A_{i}, B_{i, \mathcal{N}_{i}}, \ldots, D_{i, d, z}$ are constant real matrices of appropriate size, and may differ for each component. Index set $\mathcal{N}_{i}$ and input $q_{\mathcal{N}_{i}}$ are defined in the next section.

In the framework presented in this paper, (2) can be augmented, without loss in generality, by arbitrary LTI uncertainty models, and constant time-delay terms to model actuator and communication network effects as demonstrated in (Rödönyi, 2017).

\subsection{Interconnection Topology}

Component $\Sigma_{i}$ may have access to $q_{0}$, assuming a longrange communication technology (leader following), and also to $q_{j}, j \in \mathcal{N}_{i} \triangleq\{i, i-1, \ldots, i-r+1\}$, of arbitrary number of near predecessors in a short communication range of maximal length $r$ (multi-predecessor following (Konduri et al., 2017)). The information received from the neighboring predecessors are collected in the compound column vector, $q_{\mathcal{N}_{i}}(t) \in \mathbb{R}^{r n_{q}}$,

$$
q_{\mathcal{N}_{i}}(t) \triangleq\left[\begin{array}{llll}
q_{i}(t)^{T} & q_{i-1}(t)^{T} \cdots & q_{i-r+1}(t)^{T}
\end{array}\right]^{T} .
$$

If $i<r$, then the elements in $q_{\mathcal{N}_{i}}(t)$ with negative index are defined to be zero vectors. The corresponding input

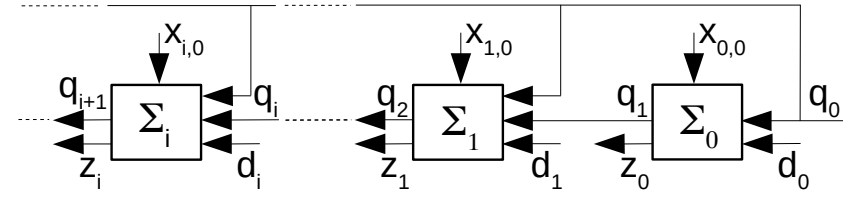

Fig. 1. Interconnection of heterogeneous dynamical systems, special case: leader and predecessor following architecture with direct neighbor communication range of $r=1$

matrices in (2) can be expanded as

$$
\left[\begin{array}{c}
B_{i, \mathcal{N}_{i}} \\
D_{i, \mathcal{N}_{i}, q} \\
D_{i, \mathcal{N}_{i}, z}
\end{array}\right] \triangleq\left[\begin{array}{cccc}
B_{i, i} & B_{i, i-1} & \cdots & B_{i, i-r+1} \\
D_{i, i, q} & D_{i, i-1, q} & \cdots & D_{i, i-r+1, q} \\
D_{i, i, z} & D_{i, i-1, z} & \cdots & D_{i, i-r+1, z}
\end{array}\right] .
$$

Note that $r$ denotes the maximum length of the limited communication range in the string. Components not utilizing all of the communication channels in this range are described by appropriate zero columns in (4). Model structure (2) covers all the LTI systems considered in the cited papers, including (cooperative) adaptive cruise control (Ploeg et al., 2014), and connected cruise control (Hajdu et al., 2016) applications. Fig. 1 illustrates the model structure for the case of a leader and predecessor following ( $L P F$ ) interconnection topology (Seiler et al., 2004; Köroğlu and Falcone, 2017), where the maximum range of communication with direct predecessors is $r=1$.

Let the sequence of initial conditions, disturbances, performance signals, and transmission signals be denoted, respectively, by $x^{0} \triangleq\left\{x_{i, 0}\right\}_{i \in \mathbb{N}}, d(t) \triangleq\left\{d_{i}(t)\right\}_{i \in \mathbb{N}}, q(t) \triangleq$ $\left\{q_{i}(t)\right\}_{i \in \mathbb{N}_{+}}, z(t) \triangleq\left\{z_{i}(t)\right\}_{i \in \mathbb{N}}$.

\subsection{Set of Components, Set of Strings}

We consider heterogeneous strings of arbitrary length and arbitrary ordering of the components. Thus we have to deal with a set of strings which is completely characterized by the set of possible components denoted by $\mathcal{S}_{\Sigma}$, i.e., $\Sigma_{i} \in \mathcal{S}_{\Sigma}$ for all $i \in \mathbb{N}$. One particular string of length $\ell$ is given by a sequence of $\ell$ interconnected components $\Omega=\left(\Sigma_{0}, \Sigma_{1}, \ldots, \Sigma_{\ell-1}\right)$, and can be viewed as an LTI system with initial states $\left\{x_{i, 0}\right\}_{i=0}^{\ell-1}$, inputs $q_{0}(t)$ and $\left\{d_{i}(t)\right\}_{i=0}^{\ell-1}$ and outputs $\left\{q_{i}(t)\right\}_{i=1}^{\ell}$ and $\left\{z_{i}(t)\right\}_{i=0}^{\ell-1}$. To express the dependence of the outputs on the inputs and on the particular string, we may write $q=q\left(\Omega, x^{0}, q_{0}, d\right)$ and $z=z\left(\Omega, x^{0}, q_{0}, d\right)$. The set of all strings of length $\ell$ is denoted by $\mathcal{S}_{\Omega}^{\ell}\left(\mathcal{S}_{\Sigma}\right) \triangleq\left\{\left(\Sigma_{0}, \Sigma_{1}, \ldots, \Sigma_{\ell-1}\right): \Sigma_{i} \in \mathcal{S}_{\Sigma}\right\}$. The set of all strings whose components are selected from set $\mathcal{S}_{\Sigma}$ is denoted by $\mathcal{S}_{\Omega}\left(\mathcal{S}_{\Sigma}\right) \triangleq \bigcup_{\ell=1}^{\infty} \mathcal{S}_{\Omega}^{\ell}\left(\mathcal{S}_{\Sigma}\right)$. The notion of string stability is associated with this set of strings, or equivalently, with the set of all possible component models $\mathcal{S}_{\Sigma}$ 


\subsection{Definitions for Heterogeneous String Stability and Performance}

The general framework proposed in this paper allows various choices for signal spaces, each may be sensible for a particular application. The following definitions require $\left(\mathcal{L}_{p_{j}}, \ell_{p_{k}}\right)$-boundedness of variables where integers $p_{1}, \ldots, p_{8} \in[1, \infty]$ refer to function spaces. Experiences on platooning applications, for instance (Naus et al., 2010, Section IV), show that different signals may have different string stability properties. The same problem is demonstrated in (Rödönyi, 2017) where, under certain circumstances, the interconnection signals (vehicle accelerations) and the performance signals (spacing errors) show different evolution properties: uniform $\mathcal{L}_{2^{-}}$ boundedness in the first, and uniform $\mathcal{L}_{\infty}$-boundedness in the latter. These problems motivate us to introduce the novel notion of string performance. By introducing appropriate scalings in the component models we may always have the following

Assumption 1 The spatial and temporal boundary conditions and the disturbances are all bounded by unity in their respective spaces, $q_{0} \in \mathcal{L}_{p_{1}}, x^{0} \in \ell_{p_{2}}$, and $d \in$ $\left(\mathcal{L}_{p_{3}}, \ell_{p_{4}}\right)$.

Definition 1 (HSS) The set of all strings $\mathcal{S}_{\Omega}\left(\mathcal{S}_{\Sigma}\right)$ that can be constructed from the set of component models, $\mathcal{S}_{\Sigma}$, is heterogeneous string stable (HSS), if the sequence of transmission signals $q\left(\Omega, x^{0}, q_{0}, d\right)$ is bounded in $\left(\mathcal{L}_{p_{5}}, \ell_{p_{6}}\right)$, i.e., $\left\|q\left(\Omega, x^{0}, q_{0}, d\right)\right\|_{p_{5}, p_{6}} \leq \gamma_{q}<\infty$, for all $\Omega \in \mathcal{S}_{\Omega}\left(\mathcal{S}_{\Sigma}\right)$ and all $\left(x^{0}, q_{0}, d\right)$ satisfying Assumption 1. Otherwise $\mathcal{S}_{\Omega}\left(\mathcal{S}_{\Sigma}\right)$ is heterogeneous string unstable.

Definition 2 (HSP) The set of all strings $\mathcal{S}_{\Omega}\left(\mathcal{S}_{\Sigma}\right)$ has heterogeneous string performance (HSP) of level $\gamma_{z}$ if the sequence of performance signals $z\left(\Omega, x^{0}, q_{0}, d\right)$ is bounded in $\left(\mathcal{L}_{p_{7}}, \ell_{p_{8}}\right)$ by $\left\|z\left(\Omega, x^{0}, q_{0}, d\right)\right\|_{p_{7}, p_{8}}<\gamma_{z}$ for all $\Omega \in$ $\mathcal{S}_{\Omega}\left(\mathcal{S}_{\Sigma}\right)$ and all $\left(x^{0}, q_{0}, d\right)$ satisfying Assumption 1.

It can be seen based on Sections 3 and 4 that (heterogeneous) string performance implies (heterogeneous) string stability, but the converse is not in general true.

The choice of space $\ell_{\infty}$ corresponds to spatially uniform boundedness of signals, a sensible assumption and requirement in most of the applications. The spatial boundary (reference) function is usually assumed to be $q_{0} \in \mathcal{L}_{2}$ or $q_{0} \in \mathcal{L}_{\infty}$ or both (Soltanian and Cantoni, $2015)$. Variables of interest, $z_{i}$, are often required to belong to $\mathcal{L}_{\infty}$ (e.g., spacing errors in platooning problems to avoid collisions). Alternative choices can be found for example in (Seiler et al., 2004; Barooah and Hespanha, 2005), where disturbances and spacing errors are assumed to be in $\left(\mathcal{L}_{2}, \ell_{2}\right)$. Except for the approaches in (D'Andrea and Dullerud, 2003) and (Knorn, 2013) there is usually no distinction between interconnection signals and performance variables in the literature.
The goal of the paper is to develop a general framework suitable for investigating HSS and HSP of unidirectionally interconnected LTI systems defined by (2).

\section{Description of Heterogeneous Strings}

The proposed method can be classified as a (frequency, space)-domain approach where Laplace-transform is applied to the component models (2)-(4), which yields the following spatially discrete parameter dependent system

$$
\left[\begin{array}{c}
q_{\mathcal{N}_{i+1}}(s) \\
\hline z_{i}(s)
\end{array}\right]=\left[\begin{array}{c|c}
\mathcal{A}_{i}(s) & \mathcal{B}_{i}(s) \\
\hline \mathcal{C}_{i}(s) & \mathcal{D}_{i}(s)
\end{array}\right]\left[\frac{q_{\mathcal{N}_{i}}(s)}{u_{i}(s)}\right],
$$

for $i \in \mathbb{N}$, where state vector $q_{\mathcal{N}_{i}}$ is defined by (3), inputs and initial conditions are collected in vector

$$
u_{i}(s) \triangleq\left[q_{0}(s)^{T}, d_{i}(s)^{T}, x_{i, 0}^{T}\right]^{T}
$$

and

$$
\begin{aligned}
& \mathcal{A}_{i}(s) \triangleq\left[\begin{array}{cccc}
\alpha_{i, i}(s) & \alpha_{i, i-1}(s) & \ldots & \alpha_{i, i-r+1}(s) \\
I & 0 & \ldots & 0 \\
& \ddots & & \vdots \\
0 & & I & 0
\end{array}\right], \\
& \alpha_{i, j}(s) \triangleq C_{i, q}\left(s I-A_{i}\right)^{-1} B_{i, j}+D_{i, j, q}, \quad j \in \mathcal{N}_{i}, \\
& \mathcal{B}_{i}(s) \triangleq C_{i, q}\left(s I-A_{i}\right)^{-1}\left[\begin{array}{ccc}
B_{i, 0}, & B_{i, d}, & I \\
0 & 0 & 0 \\
\vdots & \vdots & \vdots \\
0 & 0 & 0
\end{array}\right] \\
& +\left[\begin{array}{ccc}
D_{i, 0, q}, & D_{i, d, q}, & 0 \\
0 & 0 & 0 \\
\vdots & \vdots & \vdots \\
0 & 0 & 0
\end{array}\right] \text {, } \\
& \mathcal{C}_{i}(s) \triangleq\left[\zeta_{i, i}(s), \zeta_{i, i-1}(s), \ldots \zeta_{i, i-r+1}(s)\right] \text {, } \\
& \zeta_{i, j}(s) \triangleq C_{i, z}\left(s I-A_{i}\right)^{-1} B_{i, j}+D_{i, j, z}, \quad j \in \mathcal{N}_{i}, \\
& \mathcal{D}_{i}(s) \triangleq C_{i, z}\left(s I-A_{i}\right)^{-1}\left[B_{i, 0}, B_{i, d}, I\right] \\
& +\left[D_{i, 0, z}, D_{i, d, z}, 0\right],
\end{aligned}
$$

where matrices with negative indexes are defined as zero matrices. Description of the heterogeneous string (5) can be interpreted as a spatially discrete state-space model parameterized by the Laplace variable $s$. The dimension 
of the state-space is $\operatorname{dim}\left(q_{\mathcal{N}_{i}}\right)=r n_{q}$. The interconnection and performance variables evolve as the unique solutions to (5),

$$
\begin{aligned}
q_{\mathcal{N}_{i}}(s) & =\Phi(s ; i, 0) q_{\mathcal{N}_{0}}(s)+\sum_{j=0}^{i-1} \Phi(s ; i, j+1) \mathcal{B}_{j}(s) u_{j}(s),(7) \\
z_{i}(s) & =\mathcal{C}_{i}(s) q_{\mathcal{N}_{i}}(s)+\mathcal{D}_{i}(s) u_{i}(s)
\end{aligned}
$$

for $i \in \mathbb{N}_{+}$, where

$$
\Phi(s ; i, j) \triangleq \begin{cases}I, & i=j \\ \mathcal{A}_{i-1}(s) \mathcal{A}_{i-2}(s) \cdots \mathcal{A}_{j+1}(s) \mathcal{A}_{j}(s), & i>j\end{cases}
$$

is the fundamental matrix function of the string.

Description (5) reveals the difference between the roles of interconnection and performance variables. The classical approach of comparing $z_{i}(s)$ with $z_{i-1}(s)$ to deduce strong string stability, as in (1), is analogous to comparing two consecutive samples of the output of a discrete linear time-varying system to deduce its absolute stability. The presented approach differs from those of the literature also in the following. Although head-totail transfer matrices (Hajdu et al., 2016) could be defined and inspected based on the closed form solution (7)-(8) to (5), string stability is deduced based on the parameter dependent state-space matrices of (5).

One practical approach to characterize the set of all possible components, $\mathcal{S}_{\Sigma}$, is to define the $s$-dependent statespace matrices, $\mathcal{A}_{i}, \mathcal{B}_{i}, \mathcal{C}_{i}, \mathcal{D}_{i}$, of (5) as elements of convex hulls of given finitely many vertex systems. For example, $\mathcal{A}_{i}(s) \in \mathcal{S}_{\mathcal{A}}(s)$, where the $s$-dependent convex set

$$
\mathcal{S}_{\mathcal{A}}(s) \triangleq \operatorname{Conv}\left\{\overline{\mathcal{A}}_{l}(s), l=1,2, \ldots, \nu\right\}
$$

is determined by given vertex systems $\overline{\mathcal{A}}_{l}(s)$. In the following we restrict our attention to some practically relevant and widely used signal spaces. We assume that $q_{0} \in \mathcal{L}_{2}, x^{0} \in \ell_{\infty}$ and $d \in\left(\mathcal{L}_{2}, \ell_{\infty}\right)$, and look for general conditions under which $z, q \in\left(\mathcal{L}_{2}, \ell_{\infty}\right)$.

\section{General Conditions for Heterogeneous String Stability and Performance}

Interconnected systems with arbitrary ordering of components can be examined analogously to switching/polytopic systems. In this section, conditions for HSS and HSP are provided in terms of the joint spectral radius (JSR) of transfer matrices.

Let $\left.\mathcal{S}_{\mathcal{A}}(\omega) \triangleq \mathcal{S}_{\mathcal{A}}(s)\right|_{s=j \omega}$. All products of length $\ell$ with factors from convex hull $\mathcal{S}_{\mathcal{A}}(\omega)$ is denoted by $\mathcal{S}_{\mathcal{A}}^{\ell}(\omega) \triangleq$ $\left\{\mathcal{A}_{k_{\ell-1}}(j \omega) \mathcal{A}_{k_{\ell-2}}(j \omega) \cdots \mathcal{A}_{k_{0}}(j \omega): \mathcal{A}_{k_{i}}(j \omega) \in \mathcal{S}_{\mathcal{A}}(\omega)\right\}$.
All products of transfer matrices from this set form the semigroup $\mathscr{A}(\omega) \triangleq \bigcup_{\ell=1}^{\infty} \mathcal{S}_{\mathcal{A}}^{\ell}(\omega)$ generated by $\mathcal{S}_{\mathcal{A}}(\omega)$.

Definition 3 (JSR function) The joint spectral radius associated with set valued map $\mathcal{S}_{\mathcal{A}}(\omega)$ is a function $\sigma_{\mathcal{A}}: \mathbb{R} \mapsto \mathbb{R}_{\geq 0}$ defined by

$$
\sigma_{\mathcal{A}}(\omega) \triangleq \lim _{\ell \rightarrow \infty} \sup \left\{\|M\|^{1 / \ell}, M \in \mathcal{S}_{\mathcal{A}}^{\ell}(\omega)\right\}
$$

where $\|$.$\| denote any matrix norm.$

The following theorem provides a sufficient condition for string instability.

Theorem 1 The interconnected system (5)-(10) is heterogeneous string unstable if there exists an $\omega^{*} \in \mathbb{R}$ where $\sigma_{\mathcal{A}}\left(\omega^{*}\right)>1$.

Proof. It is shown that there exists a sequence of admissible components characterized by the sequence of matrices $\left\{\mathcal{A}_{i}\left(j \omega^{*}\right) \in \mathcal{S}_{\mathcal{A}}\left(\omega^{*}\right), i \in \mathbb{N}\right\}$ such that the sequence $\left\{\left|q_{\mathcal{N}_{i}}\left(j \omega^{*}\right)\right|, i \in \mathbb{N}\right\}$ is unbounded for any $q_{\mathcal{N}_{0}} \in \mathcal{L}_{2}$ with $\left|q_{\mathcal{N}_{0}}\left(j \omega^{*}\right)\right|>0$. By the equivalence of vector norms over finite dimensional spaces |.| may denote any vector norm. Set $\mathcal{S}_{\mathcal{A}}\left(\omega^{*}\right)$ is compact by assumption. Suppose temporarily that it is also commonly irreducible (Jungers, 2009, Section 1.2.2.5). Then there exists a Barabanov norm |.| by (Jungers, 2009, Section 2.1) such that for all $q_{\mathcal{N}_{0}}\left(j \omega^{*}\right) \in \mathbb{C}^{r n_{q}}$ there exists a matrix $\mathcal{A}_{0}\left(j \omega^{*}\right) \in \mathcal{S}_{\mathcal{A}}\left(\omega^{*}\right)$ such that $\left|\mathcal{A}_{0}\left(j \omega^{*}\right) q_{\mathcal{N}_{0}}\left(j \omega^{*}\right)\right|=\sigma_{\mathcal{A}}\left(\omega^{*}\right)\left|q_{\mathcal{N}_{0}}\left(j \omega^{*}\right)\right|$. By iterating the construction one gets an unbounded trajectory with $\left|q_{\mathcal{N}_{i}}\left(j \omega^{*}\right)\right|=\sigma_{\mathcal{A}}\left(\omega^{*}\right)^{i}\left|q_{\mathcal{N}_{0}}\left(j \omega^{*}\right)\right|$. If set $\mathcal{S}_{\mathcal{A}}\left(\omega^{*}\right)$ is commonly reducible then there exists a coordinate transformation $T \in \mathbb{C}^{r n_{q} \times r n_{q}}$ such that $T \mathcal{S}_{\mathcal{A}}\left(\omega^{*}\right) T^{-1}$ is block triangular with each diagonal block irreducible and one of these blocks has JSR equal to $\sigma_{\mathcal{A}}\left(\omega^{*}\right)>1$. The above construction can be applied to this block.

Typical LPF control structures in vehicle platooning (Seiler et al., 2004; Köroğlu and Falcone, 2017) satisfy the conditions of the next theorem which provides sufficient conditions for HSS based on the following fact. A switching system under arbitrary switching sequence, or alternatively a system with time-varying polytopic uncertainty, is asymptotically/exponentially/BIBO stable if, and only if the JSR of the set of system matrices is less than one (Michaletzky and Gerencsér, 2002; Jungers, 2009).

Theorem 2 Let $q_{0} \in \mathcal{L}_{2}, x^{0} \in \ell_{\infty}$ and $d \in\left(\mathcal{L}_{2}, \ell_{\infty}\right)$. Assume that all components satisfy the following bounded input constraint. There exists a constant $\delta$ such that $\left\|\mathcal{B}_{i} u_{i}\right\|_{2} \leq \delta$ for all $i \in \mathbb{N}$, i.e., every component $\Sigma_{i}$ has finite gain from $u_{i}$ to output $q_{\mathcal{N}_{i+1}}$. The interconnected system (5)-(10) is heterogeneous string stable, i.e., $q \in\left(\mathcal{L}_{2}, \ell_{\infty}\right)$, if $\sigma_{\mathcal{A}}(\omega)<1$ for all $\omega \in \mathbb{R}$. 
Proof. From (7) $q_{\mathcal{N}_{i}}$ is bounded by

$\left\|q_{\mathcal{N}_{i}}\right\|_{2} \leq\|\Phi(. ; i, 0)\|_{\infty}\left\|q_{\mathcal{N}_{0}}\right\|_{2}+\left\|\sum_{j=0}^{i-1} \Phi(. ; i, j+1) \mathcal{B}_{j} u_{j}\right\|_{2}$,

where the second term is bounded by $\left(\sum_{j=0}^{i-1}\|\Phi(. ; i, j+1)\|_{\infty}\right) \delta . \quad$ Observing that $\Phi(j \omega ; i, j) \in \mathcal{S}_{\mathcal{A}}^{i-j}(\omega)$ and applying (Jungers, 2009, Theorem 2.1) there is a constant $K$ such that $\bar{\sigma}(\Phi(j \omega ; i, j)) \leq K \sigma_{\mathcal{A}}(\omega)^{i-j}$. Since $\sigma_{\mathcal{A}}(\omega)<1$ for every $\omega$, the above series is finite for all $i$ and so $\left\|q_{\mathcal{N}_{i}}\right\|_{2}$ is uniformly bounded in the spacial variable $i$, i.e., $q \in\left(\mathcal{L}_{2}, \ell_{\infty}\right)$.

Typical predecessor following (PF) control structures, such as ACC and CACC in vehicle platooning, or control of irrigation channels, satisfy the inequality $\sigma_{\mathcal{A}}(\omega)<1$ only for nonzero frequencies. For accurate steady state tracking PF structures require $\sigma_{\mathcal{A}}(0)=1$. In order to ensure string stability the bounded inputs have to satisfy further constraints (for example $\mathcal{B}_{i}(s)$ must have two zeros at $s=0$ ).

Theorem 3 Let $q_{0} \in \mathcal{L}_{2}, x^{0} \in \ell_{\infty}$ and $d \in\left(\mathcal{L}_{2}, \ell_{\infty}\right)$. Assume that $\sigma_{\mathcal{A}}(0)=1, \sigma_{\mathcal{A}}(\omega)<1$ for all $\omega>0$ and there exists a common nonnegative function $\delta: \mathbb{R} \mapsto$ $\mathbb{R}_{\geq 0}, \delta \in \mathcal{L}_{2}$ with the property $\delta_{\text {max }} \triangleq \max _{\omega \in \mathbb{R}} \frac{\delta(\omega)}{\omega^{2}}<\infty$ such that

$$
\left|\mathcal{B}_{i}(j \omega) u_{i}(j \omega)\right| \leq \delta(\omega) \text { for all } i \in \mathbb{N} \text { and } \omega \in \mathbb{R}
$$

Then the interconnected system (5)-(10) is heterogeneous string stable, i.e., $q \in\left(\mathcal{L}_{2}, \ell_{\infty}\right)$.

Proof. The uniform boundedness of the first term in (12) can be proved similarly as done in the proof of Theorem 2 . Thus, there is a constant $K$ such that $\bar{\sigma}(\Phi(j \omega ; i, 0)) \leq$ $K \sigma_{\mathcal{A}}(\omega)^{i} \leq K$ for all $\omega \in \mathbb{R}$. By Parseval's theorem and by the symmetry of Fourier transform of real functions, the second term in (12) is the square root of

$$
\frac{1}{\pi} \int_{0}^{\infty}\left|\sum_{j=0}^{i-1} \Phi(j \omega ; i, j+1) \mathcal{B}_{j}(j \omega) u_{j}(j \omega)\right|^{2} d \omega
$$

To prove the finiteness of (14), we show the finiteness of the integral over the intervals $[1, \infty)$ and $[0,1]$ separately.

$$
\begin{gathered}
\int_{1}^{\infty}\left|\sum_{j=0}^{i-1} \Phi(j \omega ; i, j+1) \mathcal{B}_{j}(j \omega) u_{j}(j \omega)\right|^{2} d \omega \\
\leq \int_{1}^{\infty} \sum_{j=0}^{i-1}\left|K \sigma_{\mathcal{A}}(\omega)^{i-j-1}\right|^{2}|\delta(\omega)|^{2} d \omega \\
\quad \leq \frac{K^{2}}{1-\max _{\omega>1} \sigma_{\mathcal{A}}^{2}(\omega)}\|\delta\|_{2}^{2},
\end{gathered}
$$

which is finite. To prove the finiteness of (14) on $\omega \in$ $[0,1]$, we first define an upper-bound for $\sigma_{\mathcal{A}}(\omega)$. Without loss of generality we can assume that there is a sufficiently small constant $\tau>0$ such that

$$
\sigma_{\mathcal{A}}(\omega) \leq \frac{1}{\sqrt{\tau^{2} \omega^{2}+1}} \text { for } \omega \in[0,1]
$$

This bound is used to bound the following integral

$$
\begin{gathered}
\int_{0}^{1}\left|\sum_{j=0}^{i-1} \Phi(j \omega ; i, j+1) \mathcal{B}_{j}(j \omega) u_{j}(j \omega)\right|^{2} d \omega \\
\quad \leq \int_{0}^{1} \sum_{j=0}^{i-1}\left|K \sigma_{\mathcal{A}}(\omega)^{i-j-1}\right|^{2}\left|\omega^{2} \frac{\delta(\omega)}{\omega^{2}}\right|^{2} d \omega \\
\quad \leq K^{2} \delta_{\max }^{2} \int_{0}^{1} \sum_{j=0}^{\infty}\left|\omega^{2} \sigma_{\mathcal{A}}(\omega)^{j}\right|^{2} d \omega \\
\leq K^{2} \delta_{\max }^{2} \int_{0}^{1}\left|\frac{\omega^{2}}{1-\frac{1}{\sqrt{\tau^{2} \omega^{2}+1}}}\right|^{2} d \omega
\end{gathered}
$$

where $\lim _{\omega \rightarrow 0} \frac{\omega^{2}}{1-\frac{1}{\sqrt{\tau^{2} \omega^{2}+1}}}$ is finite by L'Hospital's rule, and so the integrand in (17) is finite everywhere. Thus, the claim is proved and $q \in\left(\mathcal{L}_{2}, \ell_{\infty}\right)$.

The following condition is sufficient in practical systems to establish the uniform boundedness of performance outputs, $z_{i}$, in the $\mathcal{L}_{2}$ norm.

Theorem 4 Suppose that the spatial and temporal boundary conditions and the disturbances satisfy $\left\|q_{0}\right\|_{2} \leq$ 1 , $\left\|x^{0}\right\|_{\infty} \leq 1$, and $\|d\|_{2, \infty} \leq 1$. The interconnected system (5)-(10) has heterogeneous string performance of level $\gamma_{z}$, i.e., $\|z\|_{2, \infty} \leq \gamma_{z}<\infty$, if both of the following conditions hold

(1) the interconnected system is $H S S$, i.e., $q \in\left(\mathcal{L}_{2}, \ell_{\infty}\right)$.

(2) $\mathcal{C}_{i}, \mathcal{D}_{i} \in \mathcal{H}_{\infty}$

Proof. From condition 1 there exists a constant $\gamma_{q}$ such that $\|q\|_{2, \infty} \leq \gamma_{q}$. From the boundedness of $\mathcal{D}_{i}$ and input $u_{i}$ defined in (6) it follows that there exists a constant $c$ such that $\left\|\mathcal{D}_{i} u_{i}\right\|_{2} \leq c$ for all $i$. Then $\|z\|_{2, \infty} \leq \gamma_{z}=$ $\max _{i}\left\|\mathcal{C}_{i}\right\|_{\infty} \gamma_{q}+c<\infty$ proves HSP of level $\gamma_{z}$.

Remark 1 HSP with $z \in\left(\mathcal{L}_{\infty}, \ell_{\infty}\right)$ can be proved similarly provided that $\mathcal{C}_{i}$ and $\mathcal{D}_{i}$ have finite generalized $\mathcal{H}_{2}$ norm, i.e., finite induced $\mathcal{L}_{2}$ to $\mathcal{L}_{\infty}$ norm.

Remark 2 The presented simple conditions for HSS, HSP or string instability can be easily tested. There are, however, systems that do not satisfy the above conditions. In these cases one may have to elaborate more specific, application dependent conditions on $\mathcal{A}_{i}, \mathcal{B}_{i}, \mathcal{C}_{i}$ and $\mathcal{D}_{i}$, or to associate more realistic sets for the inputs. One such example is presented in (Rödönyi, 2017). 


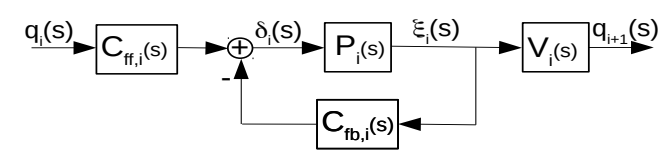

Fig. 2. The $q_{i} \stackrel{\mathcal{A}_{i}}{\mapsto} q_{i+1}$ part of component model $\Sigma_{i}$, where transfer matrix $\mathcal{A}_{i}(s)$ is of rank one for almost all $s$, control input $\delta_{i}(s)$ is scalar, interconnection variable $q_{i}(s)$ is vector.

\section{Special Problems}

It can be seen from Theorems 2 and 3 that whenever $\mathcal{B}_{i}$ satisfies some structural requirement, then HSS depends on the JSR of transfer matrices $\mathcal{A}_{i}$. Although the exact computation of the JSR for general set of matrices is theoretically NP hard, a number of approximation algorithms exist with remarkable accuracy (Jungers, 2009). In addition, the transfer matrices in most of the cited applications have special structural properties that make the computation of the JSR extremely efficient. Two practically important cases are discussed in this section.

\subsection{Scalar Interconnection Variables}

Corollary 1 For SISO transfer functions $\mathcal{A}_{i}(s) \in$ $\mathcal{S}_{\mathcal{A}}(s)$ we have $\sigma_{\mathcal{A}}(\omega)=\max _{l \in\{1, \ldots, \nu\}}\left|\overline{\mathcal{A}}_{l}(j \omega)\right|$, where $\overline{\mathcal{A}}_{l}$ and $\mathcal{S}_{\mathcal{A}}$ are defined by (10).

Corollary 1 applies to a wide range of applications. It implies that conditions for homogeneous and heterogeneous string stability are equivalent, i.e., every component can be designed for homogeneous string stability without regard to properties of other components, and when different such components are joining in arbitrary order, the string will be heterogeneous string stable.

\subsection{Rank One Systems}

Component $\Sigma_{i}$ is said to be a rank one system, if matrix $\mathcal{A}_{i}(s)$ in (5) has rank one for almost all $s \in \mathbb{C}$. Interconnected rank one systems arise, for example, when only one direct predecessor is followed (i.e., the communication range is $r=1$ ), the plant $P_{i}$ to be controlled in component $\Sigma_{i}$ is a single input multiple output system, $\xi_{i}(s)=P_{i}(s) \delta_{i}(s), P_{i}(s) \in \mathbb{C}^{\operatorname{dim}\left(\xi_{i}\right) \times 1}$, and the interconnection variable $q_{i}(s)=V_{i}(s) \xi_{i}(s) \in \mathbb{C}^{n_{q}}$ is a vector $\left(n_{q}>1\right)$, and $V_{i}(s)$ is a transfer function of rank $n_{q}$ for almost all $s \in \mathbb{C}$. A general setup is shown in Fig. 2, where performance signals, initial conditions and the possible link to the leader are omitted for simplicity. The associated interconnection transfer matrix $\mathcal{A}_{i}(s)=\frac{V_{i}(s) P_{i}(s) C_{f f, i}(s)}{1+C_{f b, i}(s) P_{i}(s)}$ mapping $q_{i}(s)$ into $q_{i+1}(s)$ can be written as a dyad $\mathcal{A}_{i}(s)=b_{i}(s) c_{i}^{T}(s)$, where $b_{i}(s)=V_{i}(s) P_{i}(s) \in \mathbb{C}^{n_{q} \times 1}$ is a column vector and $c_{i}^{T}(s)=\frac{C_{f f, i}(s)}{1+C_{f b, i}(s) P_{i}(s)} \in \mathbb{C}^{1 \times n_{q}}$ is a row vector.

The computation of the JSR of a finite set of rank one matrices is equivalent to the problem of computing the maximum cycle mean (MCM) in a directed graph. The JSR is achieved by the spectral radius of the product of at most $\nu$ different matrices (Ahmadi and Parrilo, 2012). Applied for the frequency dependent problem of interconnected systems, this means that the JSR function (11) associated with the set of transfer matrices $\mathcal{S}_{\mathcal{A}}(\omega)$ defined by (10) equals to the maximum of the spectral radii of products of at most $\nu$ vertexes of the polytope $\mathcal{S}_{\mathcal{A}}(\omega)$, i.e.,

$$
\sigma_{\mathcal{A}}(\omega)=\max _{M \in \mathscr{M}_{\nu}(\omega)} \rho(M)^{1 / \ell(M)},
$$

where $\mathscr{M}_{\nu}(\omega) \triangleq \bigcup_{\ell=1}^{\nu} \mathscr{M}^{\ell}(\omega)$ with $\mathscr{M}^{\ell}(\omega) \triangleq$ $\left\{\prod_{l=1}^{\ell} \overline{\mathcal{A}}_{k_{l}}(j \omega), k_{l} \in\{1, \ldots, \nu\}\right\}$, and $\ell(M)$ is the number of factors in $M$. Instead of testing all possible products, the MCM algorithm by Karp (Karp, 1978) provides a more efficient computation of the JSR in $\mathcal{O}\left(\nu^{3}+\nu^{2} n_{q}\right)$.

Given the set of possible components $\mathcal{S}_{\Sigma}$ defined by (5) and (10), the computation of JSR as described above can be applied as an efficient tool for the analysis of HSS. In order to derive distributed synthesis conditions, we can start from (18) observing that the spectral radius of the product of $\nu$ rank one matrices reveals the form (dependence on $\omega$ is omitted for brevity)

$$
\begin{aligned}
\rho\left(\mathcal{A}_{1} \mathcal{A}_{2} \cdots \mathcal{A}_{\nu}\right) & =\rho\left(b_{1} c_{1}^{T} b_{2} c_{2}^{T} b_{3} \cdots c_{\nu-1}^{T} b_{\nu} c_{\nu}^{T}\right) \\
& =\left|c_{\nu}^{T} b_{1}\right|\left|c_{1}^{T} b_{2}\right|\left|c_{2}^{T} b_{3}\right| \cdots\left|c_{\nu-1}^{T} b_{\nu}\right| .
\end{aligned}
$$

A sufficient condition for $\sigma_{\mathcal{A}}(\omega)<1$ to hold is that

$\sigma_{R S S}(\omega) \triangleq \max _{i, j \in\{1, \ldots, \nu\}}\left|c_{i}^{T}(\omega) b_{j}(\omega)\right|<1$.

Inequality (20) is called Robust String Stability (RSS) Condition for rank one systems. To design the control system $C_{f f, i}(s), C_{f b, i}(s)$ of component $i$ it is sufficient to satisfy (20) robustly against the set of all possible plant models $b_{j}(s)=V_{j}(s) P_{j}(s)$, and the design is independent on $c_{j}^{T}(s)$, i.e., the control systems of other components. It follows from (18) that the RSS condition guarantees strong string stability in the interconnection variables.

\section{Example: Cooperative Adaptive Cruise Con- trol (CACC) Problem}

The goal of this section is to demonstrate the power of the presented approach in analyzing HSS and HSP of interconnected MIMO LTI rank one systems.

\subsection{Component Modeling and Notes on Classical Frequency-Domain Approaches}

The following CACC system model is borrowed from (Ploeg et al., 2015). The vehicle dynamics at the $i$ th 
position of the string is described in the Laplacedomain by $a_{i}(s)=P_{i}(s) \delta_{i}(s)$, where $a_{i}$ is the vehicle acceleration, $\delta_{i}$ is the control input and $P_{i}(s)=$ $\frac{1}{\tau_{i} s+1} e^{-\phi_{i} s}$ is the transfer function of the acceleration dynamics with time constant $\tau_{i}$ and time delay $\phi_{i}$. Let $h_{i}$ denote the time-headway parameter for a constant time-headway spacing policy and introduce $H_{i}(s) \triangleq h_{i} s+1$. The spacing error is defined by $e_{i}(s) \triangleq \frac{1}{s^{2}}\left(a_{i-1}(s)-H_{i}(s) a_{i}(s)\right)$. The control structure uses control input of the predecessor vehicle: $\delta_{i}(s)=$ $H_{i}^{-1}(s)\left(K_{e, i}(s) e_{i}(s)+K_{\delta, i}(s) e^{-\theta_{i} s} \delta_{i-1}(s)\right)$, where $\theta_{i}$ denotes time-delay due to communication. Dynamic controllers $K_{e, i}(s)=k_{e, i} \frac{s-z_{e, i}}{s-p_{e, i}}$ and $K_{\delta, i}(s)=k_{\delta, i}$ are parameterized by $k_{e, i}, k_{\delta, i}>0$ and $z_{e, i}, p_{e, i}<0$.

According to the approach of this paper, a component model should depend on the parameters of a single vehicle. Consequently, the transmission signal in this example is a vector, $q_{i}(s) \triangleq\left[a_{i-1}(s), \delta_{i-1}(s)\right]^{T}$. For the performance output the spacing error is a natural choice, $z_{i}(s) \triangleq e_{i}(s)$. For brevity of the presentation, assume zero initial conditions and zero disturbances. The above CACC model then leads to $\left[\frac{q_{i+1}(s)}{z_{i}(s)}\right]=\left[\frac{\mathcal{A}_{i}(s)}{\mathcal{C}_{i}(s)}\right] q_{i}(s)$, where $\mathcal{A}_{i}(s)=$ $b_{i}(s) c_{i}^{T}(s)$ is a rank one matrix with $b_{i}(s) \triangleq\left[P_{i}(s), 1\right]^{T}$, $c_{i}^{T}(s) \triangleq\left[\frac{H_{i}^{-1}(s) K_{e, i}(s) / s^{2}}{1+K_{e, i}(s) P_{i}(s) / s^{2}}, \frac{H_{i}^{-1}(s) K_{\delta, i}(s) e^{-\theta_{i} s}}{1+K_{e, i}(s) P_{i}(s) / s^{2}}\right]$, and $\mathcal{C}_{i}(s) \triangleq\left[\frac{1}{s^{2}+K_{e, i}(s) P_{i}(s)}, \frac{P_{i}(s) K_{\delta, i}(s) e^{-\theta_{i} s}}{s^{2}+K_{e, i}(s) P_{i}(s)}\right]$.

Remark 3 If acceleration is communicated instead of control input then the same control structure $\delta_{i}(s)=$ $H_{i}^{-1}(s)\left(K_{e, i}(s) e_{i}(s)+K_{\delta, i}(s) e^{-\theta_{i} s} a_{i-1}(s)\right)$ results in $a$ scalar problem with $q_{i}(s) \triangleq a_{i-1}(s)$, and the design for homogeneous string stability always results in HSS.

Remark 4 String stability transfer functions (1) with respect to $\delta_{i}$ are obtained by inserting $a_{i-1}(s)=$ $P_{i-1}(s) \delta_{i-1}(s)$ into $q_{i}(s)$, so that $\delta_{i}(s)=\Gamma_{i}(s) \delta_{i-1}(s)$ is with $\Gamma_{i}(s)=c_{i}^{T}(s) b_{i-1}(s)$; and $\delta_{i}(s)=\Gamma_{i}^{\prime}(s) \delta_{0}(s)$ with $\Gamma_{i}^{\prime}(s)=c_{i}^{T}(s) \mathcal{A}_{i-1}(s) \mathcal{A}_{i-2}(s) \cdots \mathcal{A}_{2}(s) b_{0}(s)$. Both $\Gamma_{i}^{\prime}$ and $\Gamma_{i}$ depend on the dynamics of multiple components, which makes the HSS analysis based on these SISO transfer functions challenging (Naus et al., 2010).

Remark 5 The classical $\mathcal{H}_{\infty}$-norm based sufficient condition for string stability is $\left\|\mathcal{A}_{i}\right\|_{\infty} \leq 1$. Since $\mathcal{A}_{i}(j 0)=$ $[1,1]^{T}[1,0]$, it follows that $\left\|\mathcal{A}_{i}\right\|_{\infty} \geq \bar{\sigma}\left(\mathcal{A}_{i}(j 0)\right)=\sqrt{2}$, and so, independently on the parameters, the $\mathcal{H}_{\infty}$-norm based condition never holds. It is shown in Section 6.2 that examining the JSR function of the set of vehicles provides a non-conservative test for $H S S$.

Remark 6 Consider the error string stability transfer function $\Gamma_{i}(s) \triangleq \frac{e_{i}(s)}{e_{i-1}(s)}$ (Naus et al., 2010). With the above notations, $\Gamma_{i}(s)=\frac{\mathcal{C}_{i}(s) \mathcal{A}_{i-1}(s) b_{i-2}(s)}{\mathcal{C}_{i-1}(s) b_{i-2}(s)}$. Static gain $\Gamma_{i}(j 0)=\frac{1+k_{\delta, i}}{1+k_{\delta, i-1}} \frac{k_{e, i-1} z_{e, i-1} p_{e, i}}{k_{e, i} z_{e, i} p_{e, i-1}}$ depend on the control parameters of two vehicles, which implies that the classical strong string stability condition $\left\|\Gamma_{i}\right\|_{\infty} \leq 1$ with respect to the spacing errors cannot be satisfied in an arbitrary heterogeneous platoon. This problem can be resolved by the distinction of transmission and performance signals and the respective notions of HSS and HSP: outputs should not be compared in this way, since the transmission variables are propagating in space, not the outputs.

\subsection{Numerical Examples}

In order to demonstrate the power of the JSR based analysis approach and the advantage of distinguishing between the HSS and HSP problems, it is sufficient to consider heterogeneous platoons where the set of possible vehicles consists of two elements, $\mathcal{S}_{\Sigma}=\left\{\Sigma_{1}, \Sigma_{2}\right\}$. Each vehicle $\Sigma_{i}$ is characterized by the pair of transfer functions $\left(\mathcal{A}_{i}(s), \mathcal{C}_{i}(s)\right)$. A heterogeneous platoon associated with the set of vehicles $\mathcal{S}_{\Sigma}$ is given by defining the sequence of the vehicles $\Omega=\left(\Sigma_{k_{0}}, \Sigma_{k_{1}}, \Sigma_{k_{2}}, \ldots\right)$, $k_{i} \in\{1,2\}$. According to Karp's algorithm the JSR of two matrices, $\mathcal{S}_{\mathcal{A}}(\omega) \triangleq\left\{\mathcal{A}_{1}(j \omega), \mathcal{A}_{2}(j \omega)\right\}$, is achieved by a sequence which is either homogeneous $\left(\mathcal{A}_{1} \mathcal{A}_{1} \mathcal{A}_{1} \ldots\right.$ or $\left.\mathcal{A}_{2} \mathcal{A}_{2} \mathcal{A}_{2} \cdots\right)$, or heterogeneous with alternating matrices $\left(\mathcal{A}_{1} \mathcal{A}_{2} \mathcal{A}_{1} \mathcal{A}_{2} \cdots\right)$. Thus, the JSR function (18) associated with $\mathcal{S}_{\mathcal{A}}(\omega)$ can be computed as the maximum of three terms

$$
\begin{aligned}
\sigma_{\mathcal{A}}(\omega)=\max & \left\{\left|c_{1}^{T}(j \omega) b_{1}(j \omega)\right|,\left|c_{2}^{T}(j \omega) b_{2}(j \omega)\right|,\right. \\
& \left.\sqrt{\left|c_{1}^{T}(j \omega) b_{2}(j \omega)\right|\left|c_{2}^{T}(j \omega) b_{1}(j \omega)\right|}\right\} .
\end{aligned}
$$

In the examples, the plant models are $P_{1}(s)=$ $\frac{e^{-0.1 s}}{0.1 s+1}$ for vehicle $\Sigma_{1}$, and $P_{2}(s)=\frac{e^{-0.145 s}}{0.35 s+1}$ for vehicle $\Sigma_{2}$. The communication delays are $\theta_{1}=\theta_{2}=$ $0.04 \mathrm{~s}$. The controller parameters are given in the form $\Sigma_{i}\left(h_{i}, k_{e, i}, k_{\delta, i}, z_{e, i}, p_{e, i}\right), i=1,2$. For each example the frequency-domain test functions (21) or (20) and simulation results with 50 vehicles are plotted in the corresponding figure. The simulations are excited by the leader vehicle's control input, $\delta_{-1}(t)=1 \mathrm{~m} / \mathrm{s}^{2}$ for $0 \leq$ $t \leq 1 s$ and $\delta_{-1}(t)=0$ for $t>1 s$.

Example $1 \mathcal{S}_{\Sigma}=\left\{\Sigma_{1}(0.387,2.128,1,-0.209,-3.162)\right.$, $\left.\Sigma_{2}(0.427,3.162,1,-0.316,-3.162)\right\}$. Platoons that can be constructed from this set of vehicles illustrate that vehicles designed for homogeneous string stability, i.e., satisfying $\left|c_{i}^{T}(j \omega) b_{i}(j \omega)\right| \leq 1$ (see $\Gamma_{i}$ in Remark 4), may be heterogeneous string unstable. The appropriate test is the JSR function (21) shown in Fig. 3. Its $0.71 \mathrm{~dB}$ peak at $\omega^{*}=1.1 \mathrm{rad} / \mathrm{s}^{2}$ implies amplifying sinusoid components of period $\frac{2 \pi}{\omega^{*}}=5.7 \mathrm{~s}$ of the propagating signals.

Example $2 \mathcal{S}_{\Sigma}=\left\{\Sigma_{1}(0.837,2.063,1,-0.208,-3.162)\right.$, $\left.\Sigma_{2}(0.398,3.562,0.999,-0.24,-4.79)\right\}$. A practical con- 

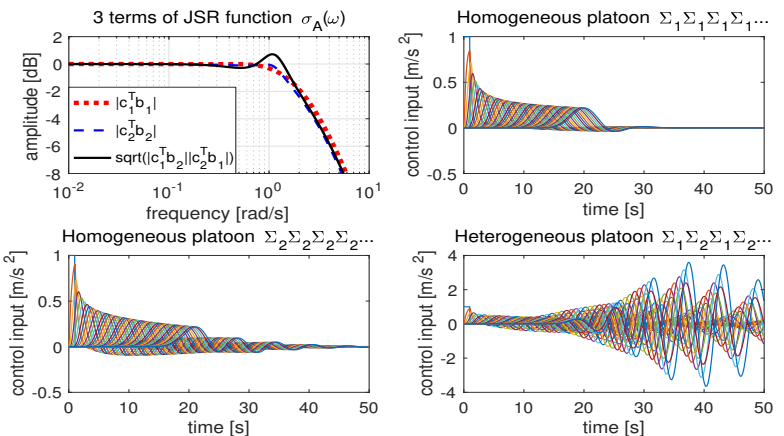

Fig. 3. Example 1 illustrates that homogeneous string stability does not imply heterogeneous string stability.

trol design method is that we try to satisfy the sufficient RSS condition (20). This task is distributed in the sense that each component has its own responsibility to contribute on HSS. RSS condition might be, however, very conservative, as shown in Fig. 4. JSR function and simulations indicate HSS of the platoons, while RSS condition fails due to a large peak at $\omega=1 \mathrm{rad} / \mathrm{s}$.

Example $3 \mathcal{S}_{\Sigma}=\left\{\Sigma_{1}(1.2,2.00,1.364,-0.196,-3.162)\right.$, $\left.\Sigma_{2}(1.2,3.44,0.873,-0.252,-4.332)\right\}$. In the previous example we have just picked up two controllers for $\Sigma_{1}$ and $\Sigma_{2}$ to illustrate the possible gap between the HSS and $R S S$ conditions. The presented CACC model structure, however, allows the RSS condition to closely approach the JSR based condition when optimizing the control parameters in order to minimize $\left\|\sigma_{R S S}\right\|_{\infty}$, see Fig. 5, where the vehicles satisfy the RSS condition (20).

Example $4 \mathcal{S}_{\Sigma}=\left\{\Sigma_{1}(1.164,2.128,1,-0.208,-3.162)\right.$, $\left.\Sigma_{2}(1.2,5.226,0.873,-0.316,-4.332)\right\}$. In the design of the previous example we focused only on achieving HSS by minimizing the RSS criterion $\left\|\sigma_{R S S}\right\|_{\infty}$. In this example, the control parameters of each vehicle are optimized to minimize the HSP criterion $\left\|\mathcal{C}_{i} b_{i}\right\|_{\infty}$ subject to the constraint $\left\|\sigma_{R S S}\right\|_{\infty} \leq 1$. As a result, the spacing errors decreased significantly in the simulations, see Fig. 6, and compare with Fig. 5. We note that applying a generalized $\mathcal{H}_{2}$ system norm in the criterion and assuming $q_{0} \in \mathcal{L}_{2}$ lead to guranteed bounds of the spacing errors in the $\mathcal{L}_{\infty}$ norm.

\section{Conclusion}

A general framework has been introduced for the analysis of signal propagation in unidirectionally interconnected string of arbitrary MIMO LTI systems. The spatial evolution of signals are described as the solutions of discrete linear state-space systems whose exponential or absolute stability properties imply analogous weak or strong notions for string stability. The necessity of distinguishing between the notions of string stability and string performance is presented. Advantages of the proposed approach over classical frequency domain meth-
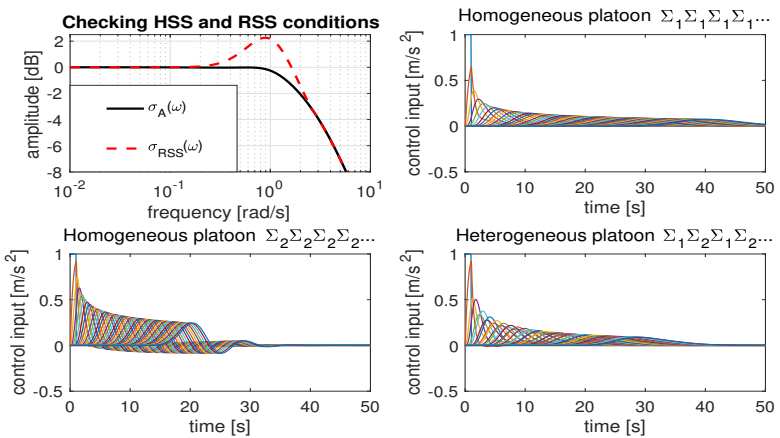

Fig. 4. Example 2 illustrates that the RSS condition (20) might be a conservative test for HSS as compared to the JSR based condition
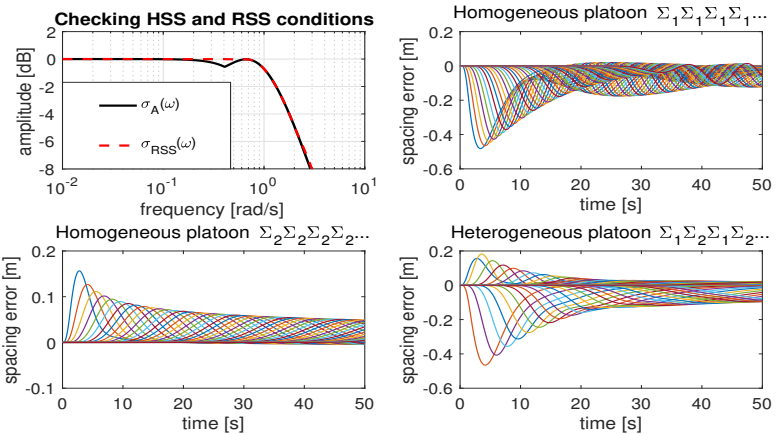

Fig. 5. Example 3 illustrates that, depending on the particular application, the RSS condition might be an appropriate tool for control synthesis
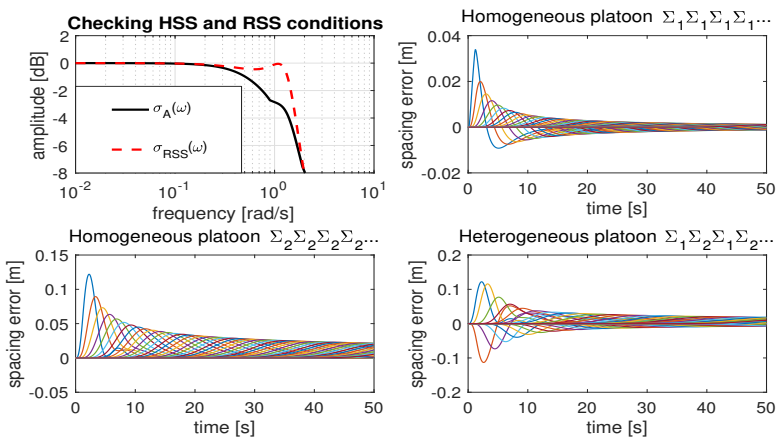

Fig. 6. Example 4 illustrates a synthesis method, where a HSP criterion is minimized subject to a HSS constraint

ods has been shown regarding heterogeneity, generality of components, simplicity in stability conditions, and handling the effects of initial conditions, reference inputs and disturbances in a unifying model.

\section{References}

Ahmadi, A. A. and Parrilo, P. A. (2012), Joint spectral radius of rank one matrices and the maximum cycle mean problem, in '51st IEEE Conf. Decision and Control', pp. 731-733.

Barooah, P. and Hespanha, J. P. (2005), Error amplifi- 
cation and disturbance propagation in vehicle strings with decentralized linear control, in '44th IEEE Conf. Decision and Control', pp. 4964-4969.

D'Andrea, R. and Dullerud, G. E. (2003), 'Distributed control design for spatially interconnected systems', IEEE Trans. Automatic Control 48(9), 1478-1495.

Hajdu, D., Zhang, L., Insperger, T. and Orosz, G. (2016), 'Robust stability analysis for connected vehicle systems', 13th IFAC Workshop on Time Delay Systems, Istanbul, Turkey pp. $165-170$.

Huang, X., Yan, N. and Guo, H. (2007), 'An $\mathrm{H}_{\infty}$ control method of the bullwhip effect for a class of supply chain system', Int. J. Production Research 45(1), 207-226.

URL: $h t t p: / / d x . d o i . o r g / 10.1080 / 00207540600678912$

Ioannou, P. A. and Chien, C. C. (1993), 'Autonomous intelligent cruise control', IEEE Trans. Vehicular Technology 42(4), 657-672.

Jungers, R. (2009), The joint spectral radius: theory and applications, Vol. 385, Springer, Lecture Notes in Control and Information Sciences.

Karp, R. M. (1978), 'A characterization of the minimum cycle mean in a digraph', Discrete Mathematics 23(3), $309-311$.

Kianfar, R., Falcone, P. and Fredriksson, J. (2011), 'A receding horizon approach for designing string stable cooperative adaptive cruise control', IEEE Conf. Intelligent Transportation Systems pp. 734-739.

Knorn, S. (2013), 'A two-dimensional systems stability analysis of vehicle platoons', PhD dissertation, Hamilton Institute, National University of Ireland, Maynooth .

Konduri, S., Pagilla, P. and Darbha, S. (2017), 'Vehicle platooning with multiple vehicle look-ahead information', 20th IFAC World Congress 50(1), 5768 - 5773.

Köroğlu, H. and Falcone, P. (2017), 'Robust static output feedback synthesis for platoons under leader and predecessor feedback', Int. J. Robust and Nonlinear Control 27, 1726-1756.

Lidstrom, K., Sjoberg, K., Holmberg, U., Andersson, J., Bergh, F., Bjade, M. and Mak, S. (2012), 'A modular CACC system integration and design', IEEE Trans. Intelligent Transportation Systems 13(3), 1050-1061.

McAree, O. and Veres, S. M. (2016), 'Lateral control of vehicle platoons with on-board sensing and intervehicle communication', European Control Conf., Aalborg, Denmark pp. 2465-2470.

Michaletzky, G. and Gerencsér, L. (2002), 'Bibo stability of linear switching systems', IEEE Trans. Automatic Control 47(11), 1895-1898.

Monteil, J., Bouroche, M. and Leith, D. J. (2018), 'L2 and $\mathrm{L}_{\infty}$ stability analysis of heterogeneous traffic with application to parameter optimization for the control of automated vehicles', IEEE Trans. Control Systems Technology pp. 1-16.

Naus, G., Vugts, R., Ploeg, J., Molengraft, M. and Steinbuch, M. (2010), 'String-stable CACC design and experimental validation: A frequencydomain approach', IEEE Trans. Vehicular Technology
59(9), 4268-4279.

Peters, A. A., Middleton, R. H. and Mason, O. (2014), 'Leader tracking in homogeneous vehicle platoons with broadcast delays', Automatica 50(1), $64-74$.

Ploeg, J., van de Wouw, N. and Nijmeijer, H. (2014), ' $\mathcal{L}_{p}$ string stability of cascaded systems: Application to vehicle platooning', IEEE Trans. Control Systems Technology 22(2), 786-793.

Ploeg, J., van de Wouw, N. and Nijmeijer, H. (2015), 'Fault tolerance of cooperative vehicle platoons subject to communication delay', 12th IFAC Workshop on Time Delay Systems, TDS 2015 - Ann Arbor, Michigan, USA 48(12), 352 - 357.

Rödönyi, G. (2017), 'An adaptive spacing policy guaranteeing string stability in multi-brand ad hoc platoons', Appears in IEEE Trans. Intelligent Transportation Systems, doi: 10.1109/TITS.2017.2749607.

Rödönyi, G. (2018), Extensions of acc technology toward ad hoc platooning with guaranteed string stability and improved tracking performance, in '50th Int. Symposium on Circuits and Systems, Florence, Italy'.

Šebek, M. and Hurák, Z. (2011), '2-d polynomial approach to control of leader following vehicular platoons', IFAC Proceedings Volumes 44(1), 6017 - 6022.

Seiler, P., Pant, A. and Hedrick, K. (2004), 'Disturbance propagation in vehicle strings', IEEE Trans. Automatic Control 49(10), 1835-1842.

Shaw, E. and Hedrick, J. (2007), String stability analysis for heterogeneous vehicle strings, in 'American Control Conf.', pp. 3118-3125.

Soltanian, L. and Cantoni, M. (2015), 'Decentralized string-stability analysis for heterogeneous cascades subject to load-matching requirements', Multidimensional Syst. Signal Process. 26(4), 985-999.

Swaroop, D. and Hedrick, J. (1996), 'String stability of interconnected systems', IEEE Trans. Automatic Control 41(3), 349-357.

Yu, B., Freudenberg, J., Gillespie, R. and Middleton, R. (2015), 'Beyond synchronization: String instability in coupled harmonic oscillator systems', Int. J. Robust and Nonlinear Control 25(15), 2745-2769. 